\title{
Wachkoma häufig falsch diagnostiziert
}

\author{
Neurophysiologische Verfahren entdecken verborgenes Bewusstsein
}

Etwa 40 Prozent aller Wachkoma-Patienten sind bei minimalem Bewusstsein, ohne dass Angehörige und das Behandlungsteam dies erkennen, und werden daher häufig nicht ausreichend therapiert.

Derzeit wird der Bewusstseinszustand mithilfe einer komplexen Skala diagnostiziert, die unter anderem visuelle und motorische Funktionen erfasst. Dieser Goldstandard wird aber noch zu selten angewendet, bemängeln Experten der Deutschen Gesellschaft für Klinische Neurophysiologie und funktionelle Bildgebung (DGKN). Zudem könnten neurophysiologische Methoden dieses Verfahren ergänzen, um Patienten mit einem minimalen Bewusstsein sicher zu identifizieren, so das Ergebnis einer Studie.

Die Zahl der Wachkomapatienten wird in Österreich mit 800 bis 1000 angegeben. Experten sprechen vom Syndrom der reaktionslosen Wachheit (SRW). Nach einer schweren Hirnschädigung öffnen sie wieder die Augen, lassen aber weder Bewusstsein noch Kontaktfähigkeit erkennen. Zeigen solche Patienten bewusste Wahrnehmungen, zum Beispiel gezielte Augenbewegungen, befinden sie sich nicht mehr im Wachkoma, sondern im sogenannten minimalen Bewusstseinszustand, auch Syndrom des minimalen Bewusstseins (SMB) genannt. In der derzeitigen klinischen Versorgungsrealität sei die Abgrenzung zwischen Wachkoma und SMB jedoch schwierig, sagt der DGKN-Experte Dr. med. Andreas Bender vom Therapiezentrum Burgau. „Studien belegen eine hohe Rate an Fehldiagnosen von bis zu 40 Prozent."

\section{Fehlende Ansprache aufgrund falscher Einschätzung}

Für die Therapie könne diese Fehldiagnose schwerwiegende Folgen haben. "Wir vermuten, dass viele SMB-Patienten, die für Wachkoma-Patienten gehalten werden, unter der fehlenden persönlichen Ansprache leiden“, so Bender. Einige werden daher verfrüht palliativ behandelt. „Die Ärzte versuchen nur noch das Leiden des vermeintlichen Wachkoma-Patienten zu reduzieren, anstatt sämtliche therapeutischen Möglichkeiten auszuschöpfen“, kritisiert der DGKN-Experte.

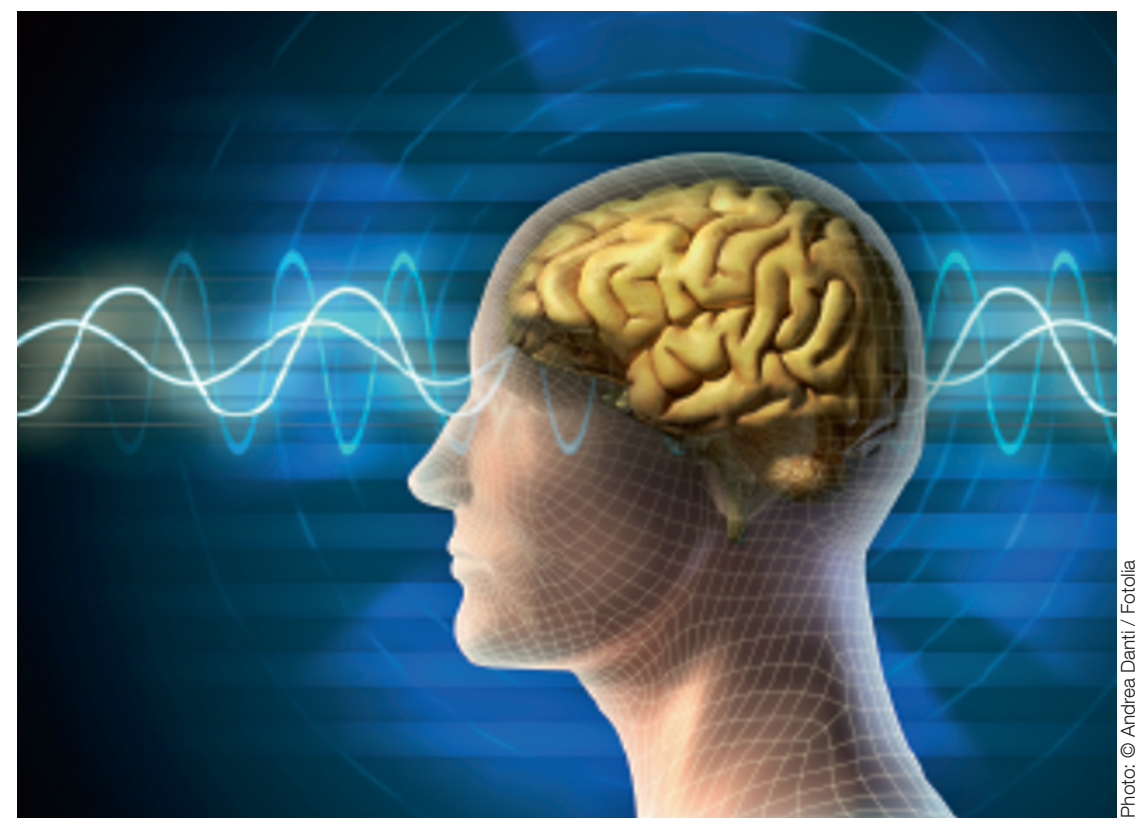

Die Messung der Hinrströme ermöglicht eine exaktere Erfassung des Bewusstseinszustands.

Mit der aktuellen Metastudie zeigt das Forscherteam um Bender, dass elektrophysiologische Verfahren solche Fehldiagnosen reduzieren können. Für die Analyse hatten die Neurophysiologen 20 klinische Studien mit 436 Wachkoma- und 470 SMB-Patienten ausgewertet, deren Hirnaktivität unter anderem mit funktioneller Magnetresonanztomographie (fMRT) und quantitativer Elektroenzephalographie (qEEG) gemessen worden war. Beide Methoden zeigen, ob Patienten sich bestimmte Bewegungen, etwa Tennisspielen, vorstellen können, wenn man sie dazu auffordert. Sowohl in den fMRT- als auch in den EEG-basierten Studien konnten zehn bis 24 Prozent der Wachkoma-Patienten solche Aufgaben befolgen. Diese Patienten wären also nicht als Wachkomapatienten, sondern als SMB-Patienten mit wenigstens partiell erhaltenem Bewusstsein einzustufen und zwar trotz der gründlichen standardisierten Untersuchung.

\section{Bessere Diagnose \\ über Elektrophysiologie}

„Mit dem derzeitigen Goldstandard für die Wachkoma-Diagnose, der sogenannten revidierten Coma Recovery Scale (CRS-R), lassen sich solche Bewegungsvorstellun- gen nicht nachweisen“, erklärt Prof. Dr. Andreas Straube vom Klinikum der Universität München die Ursache der Fehldiagnosen. Denn die CRS-R erfordert, dass Patienten sich tatsächlich bewegen. Dennoch bleibt diese klinische Einstufung derzeit der Goldstandard der Diagnostik.

„Es gibt aber auch Patienten, die zwar bei Bewusstsein sind, aber keinen Zugriff auf ihr motorisches System haben", so Bender. Besser geeignet seien für diese $\mathrm{Pa}$ tienten möglicherweise neurophysiologische Verfahren. „Am genauesten konnte der Bewusstseinszustand mit dem qEEG erkannt werden“, erklärt der DGKN-Experte die aktuellen Ergebnisse. Um diese Methode korrekt anzuwenden, bedarf es jedoch einer speziellen Ausbildung.

Quelle: Presseaussendung Deutsche Gesellschaft für Klinische Neurophysiologie und funktionelle Bildgebung (DGKN)/Bender et al. „,Wachkoma und minimaler Bewusstseinszustand: Systematisches Review und Metaanalyse zu diagnostischen Verfahren“, Dtsch Arztebl Int 2015; 112(14): 235-42; DOI: 10.3238/arztebl.2015.0235n

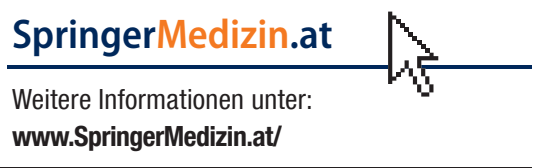

